\title{
High expression of ALDOA and DDX5 are associated with poor prognosis in human colorectal cancer
}

This article was published in the following Dove Press journal:

Cancer Management and Research

\author{
Ling Dai ${ }^{1, *}$ \\ Guangdong Pan ${ }^{2, *}$ \\ Xiaojia Liu $^{3}$ \\ Jiang Huang ${ }^{4}$ \\ Zhiqing Jiang' \\ Xiaobao Zhu' \\ Xinli Gan' \\ Qing $X u^{5}$ \\ Ning $\operatorname{Tan}^{5}$
}

'Department of Gastroenterology Surgery, Affiliated Hospital of Guilin Medical University, Guilin, People's Republic of China; ${ }^{2}$ Department of Hepatobiliary Surgery, The People's Hospital of Liuzhou, Liuzhou, People's Republic of China; ${ }^{3}$ Department of Pathology, Shanghai Medical College, Fudan University, Shanghai, People's Republic of China; ${ }^{4}$ Institute of Biotechnology, Guilin Medical University, Guilin, People's Republic of China; ${ }^{5}$ Laboratory of Liver Injury and Repair Molecular Medicine, Guilin Medical University, Guilin, People's Republic of China

*These authors contributed equally to this work

Correspondence: Ning Tan Laboratory of Liver Injury and Repair Molecular Medicine, Guilin Medical University, 109 HuanchengBei Road, Guilin 54I00I, Guangxi, People's Republic of China

Tel +86 7732809503

Fax +86 7732822703

Email windl46wind@aliyun.com
Purpose: The identification of prognostic markers for colorectal cancer (CRC) is needed for clinical practice. Fructose-bisphosphate aldolase A (ALDOA) and DEAD box p68 RNA helicase (DDX5) are commonly overexpressed in cancer and correlate with tumorigenesis. However, association between expression of ALDOA and DDX5, and CRC outcome has not been reported. Patients and methods: We used 141 formalin-fixed paraffin-embedded (FFPE) specimens collected from 105 patients with CRC treated at the Affiliated Hospital of Guilin Medical University and the People's Hospital of Liuzhou. We performed tissue microarray based immunohistochemistry to explore expression features and prognostic value (overall survival, OS; disease-free survival, [DFS]) of ALDOA and DDX5 in CRC tissues. The prognostic values were evaluated using Kaplan-Meier analysis, and Cox regression analyses.

Results: ALDOA and DDX5 were highly expressed in CRC tissues and liver metastatic CRC tissues compared with normal glandular epithelium tissues (all $p<0.05$ ). Interestingly, primary CRC tissues highly expressing ALDOA or DDX5 had poor outcome $(p<0.0001$ for both OS and DFS for ALDOA; $p=0.001$ for OS; and $p=0.011$ for DFS for DDX5) compared with patients who had low expression of those proteins. Furthermore, multivariate Cox analysis showed that ALDOA/DDX5 combination was an independent risk factor for OS and ALDOA was an independent risk factor for DFS.

Conclusion: High levels of ALDOA and DDX5 contribute to the aggressiveness and poor prognosis of CRC. ALDOA/DDX5 expression could be a biomarkers for the prognosis of CRC. Keywords: fructose-bisphosphate aldolase A, DEAD box p68 RNA helicase, colorectal cancer, $\mathrm{X}$-tile, overall survival, disease free survival

\section{Introduction}

Colorectal cancer (CRC) is a common malignancy of the digestive system and ranks fifth in China for both cancer incidence (376.3 per 100,000) and mortality (191.0 per 100,000). ${ }^{1}$ Over the past decade, significant progress has been made in the treatment of CRC through advances in surgery, radiotherapy, chemotherapy, and targeted therapy. ${ }^{2-4}$ However, the majority of patients are diagnosed at an advanced stage, limiting the therapeutic options for improving the survival rate and leading to a poor prognosis. ${ }^{5}$ In recent years, several prognostic markers such as SERPINA $4,{ }^{6}$ c-MYC, and $\beta$-catenin ${ }^{7}$ have been proposed as immunohistochemical markers for $\mathrm{CRC}$, while established diagnostic and prognostic serum biomarkers, including carbohydrate antigen 19-9 (CA19-9), ${ }^{8}$ are of value in the treatment of CRC patients. However, the need remains to identify effective biomarkers that can classify patients at high or low risk of outcomes after surgical resection. 
Fructose-bisphosphate aldolase A (ALDOA), an aldolase isozyme, plays a key role in glycolysis and gluconeogenesis ${ }^{9}$ and is highly expressed in many types of cancers, including kidney, lung, ${ }^{10}$ oral squamous cell, ${ }^{11}$ and hepatocellular carcinomas. ${ }^{12}$ DEAD box p68 RNA helicase (DDX5) is considered a prototypic member of the DEAD-box family of RNA helicases. ${ }^{13}$ Recent studies have also demonstrated that DDX5 is aberrantly expressed in several types of cancers, including colon cancer, ${ }^{14}$ breast cancer, ${ }^{15}$ lung cancer, ${ }^{16}$ cutaneous squamous cell carcinoma, ${ }^{17}$ and suggesting that DDX5 plays important roles in cancer development and progression. ${ }^{18}$ However, limited information was available for prognostic value of ALDOA and DDX5 in CRC.

In this study, we demonstrated that ALDOA is highly expressed in CRC and liver metastatic CRC tissues compared with paired normal glandular epithelium tissues, and could therefore represent a potential prognostic marker for primary CRC patients after surgical resection.

\section{Materials and methods Patient tissue samples}

We evaluated 141 formalin-fixed paraffin-embedded (FFPE) specimens (105 CRC tissues, 18 paired normal glandular epithelium tissues, and 18 liver metastatic CRC tissues) collected from 105 patients with CRC treated at the Affiliated Hospital of Guilin Medical University and the People's Hospital of Liuzhou from April 2012 to March 2015 with written informed consent by each patient and approval from the institutional review board of Affiliated Hospital of Guilin Medical University and the People's Hospital of Liuzhou was obtained. H\&E-stained slides were prepared from each FFPE specimen and were reviewed by experienced pathologists. The diagnosis of CRC was confirmed based on clinical manifestation, and pathological and serological examinations.

\section{Follow-up}

Overall survival (OS) was calculated from the date of surgery to the date of death or the last known follow-up. Diseasefree survival (DFS) was calculated from the date of tumor resection until the detection of tumor recurrence, metastasis, or death. All patients enrolled in this study provided written informed consent and the protocol was approved by the ethics committees of the Affiliated Hospital of Guilin Medical University and the People's Hospital of Liuzhou. No patients had received radiotherapy, chemotherapy, hormone therapy, or other related anti-tumor therapies prior to surgery. Follow-up data were summarized at the end of October 2016. Follow-up of all of the patients was carried out at both hospitals, including tumor marker testing every 3 months and diagnostic imaging at least every 6 months, based on the surveillance suggested in the guidelines. In cases of suspected recurrence, MRI or CT were included in the diagnostic imaging. ${ }^{19}$

\section{Tissue microarrays and immunohistochemistry}

Tissue microarrays were constructed from a representative core from each FFPE specimen. Tissue cylinders with a diameter of $1.5 \mathrm{~mm}$ were punched from marked areas of each sample and incorporated into a recipient paraffin block. Sections of $4 \mu \mathrm{m}$ thickness were placed on slides coated with 3-aminopropyltriethoxysilane. All H\&E-stained slides were reviewed by experienced pathologists and the representative cores were pre-marked in the paraffin blocks. ${ }^{20}$

Paraffin sections were deparaffinized in xylene and rehydrated using decreasing concentrations of ethanol (100\%, $95 \%$, and $85 \%$ for $5 \mathrm{~min}$ each). Antigen retrieval was performed by microwave irradiation for $5 \mathrm{~min}$ in $\mathrm{pH}$ 6.0 citric buffer, after which the samples were cooled at room temperature for $60 \mathrm{~min}$. Endogenous peroxidase activity was blocked by incubation of the slides in $3 \% \mathrm{H}_{2} \mathrm{O}_{2} /$ phosphate-buffered saline, and non-specific binding sites were blocked with goat serum. ${ }^{20}$ Mouse monoclonal antibody to ALDOA (H00000226-M02, Abnova, Taipei City, Taiwan; 1:200 dilution) and rabbit monoclonal antibody to DDX5 (ab126730, Abcam, Cambridge, UK; 1:400 dilution) were used as a primary antibody, and an EnVision Detection Kit (GK500705: Gene Tech, Shanghai, People's Republic of China) was used to visualize tissue antigens. Tissue sections were counterstained with hematoxylin for $5 \mathrm{~min}$. Negative control slides without primary antibody were prepared for all assays. Imaging was performed using a Leica CCD DFC420 camera connected to a Leica DM IRE2 microscope (Leica Microsystems Imaging Solutions Ltd, Cambridge, UK). Photographs of representative fields were captured under high-power magnification $(\times 200)$ using Leica QWin Plus v3 software. The integrated optical density (IOD) of each image was measured using Image-Pro Plus v6.0 software (Media Cybernetics Inc, Bethesda, MD, USA). ${ }^{20}$ Expression levels of ALDOA and DDX5 were thus represented by IOD. ${ }^{20}$

\section{Statistical analysis}

X2 analyses, correlations between variables, Kaplan-Meier analyses, univariate survival analysis, and multiple Cox proportional hazards regression were performed using the SPSS statistical software package (SPSS Standard version 13.0; 
SPSS, Chicago, IL, USA). The optimum cut-off points for ALDOA and DDX5 expression were obtained using X-tile software version 3.6.1 (Yale University School of Medicine, New Haven, CT, USA) to determine the relationship between protein expression and clinical outcomes (OS and DFS). ${ }^{21}$ A significant difference was considered if the $p$-value from a two-tailed test was $<0.05$.

\section{Results}

\section{Expression levels of ALDOA and DDX5 in paired adjacent glandular epithelium, CRC tumor tissues, and liver metastatic CRC tissues}

ALDOA expression in adjacent glandular epithelium was negative or low (Figure 1A), but high expression was observed in CRC tumor tissue (Figure 1B) and liver metastatic CRC tissue (Figure 1C). Significant statistical differences were observed between the paired adjacent normal glandular epithelium tissue and the CRC tumor tissue $(n=18$, $p=0.0001$, Figure 1D), and also between the paired adjacent normal glandular epithelium tissue and the liver metastatic CRC tissue $(n=18, p<0.0001$, Figure 1D). In addition, ALDOA was highly expressed in liver metastatic CRC tissue compared with paired primary CRC tissue $(p=0.0769)$. In a parallel manner, DDX5 expression was significantly higher in CRC tumor tissue (Figure $1 \mathrm{~F} ; \mathrm{n}=18, p=0.0034$, Figure $1 \mathrm{H}$ ) and liver metastatic CRC tissue (Figure $1 \mathrm{G} ; \mathrm{n}=18, p=0.0087$, Figure 1H) compared with tissues of adjacent glandular epithelium (Figure 1E, H).

\section{High expression of ALDOA and DDX5 are associated with poor prognosis}

According to the optimum cut-off point obtained from X-tile analyses, OS and DFS were estimated by Kaplan-Meier analyses. Forty-six patients were classified in the high ALDOA expression group and 59 were classified in the low ALDOA expression group; 83 patients were classified in the low DDX5 expression group and 22 in the high expression group. Kaplan-Meier analyses clearly showed that CRC patients with higher expression of ALDOA had worse OS and DFS compared with patients with lower expression of ALDOA ( $p<0.0001$ for both OS and DFS; Figure 2A, B); higher expression of DDX5 had worse OS and DFS, compared with patients with lower expression of DDX5 ( $p=0.001$ for OS and $p=0.011$ for DFS; Figure $2 \mathrm{C}, \mathrm{D})$. Notably, when combining ALDOA and DDX5, the prognostic values were more significant for predicting OS and DFS (Figure 2E, F).

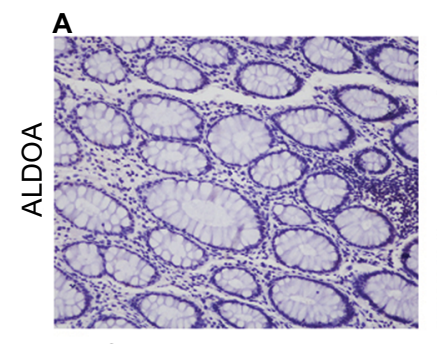

Glandular epithelium

E

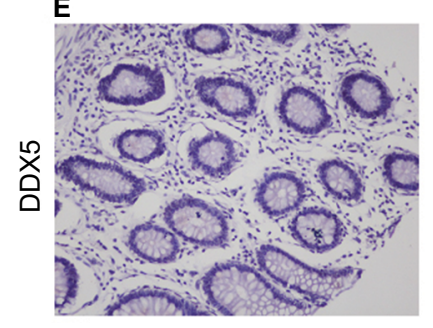

Glandular epithelium

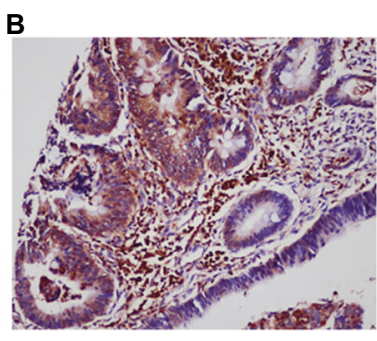

CRC

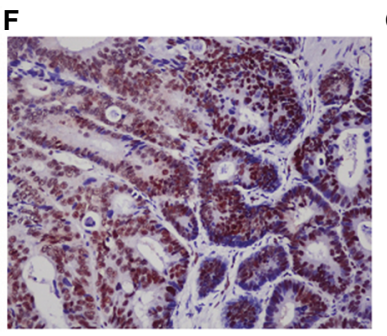

$\mathrm{CRC}$

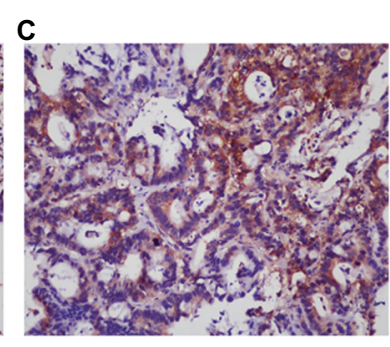

Liver metastastic CRC

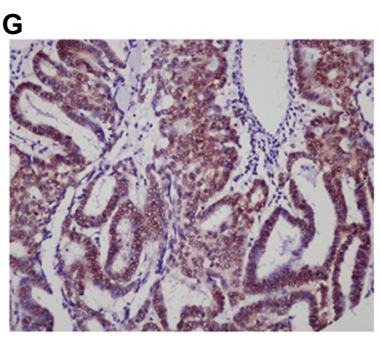

Liver metastastic CRC
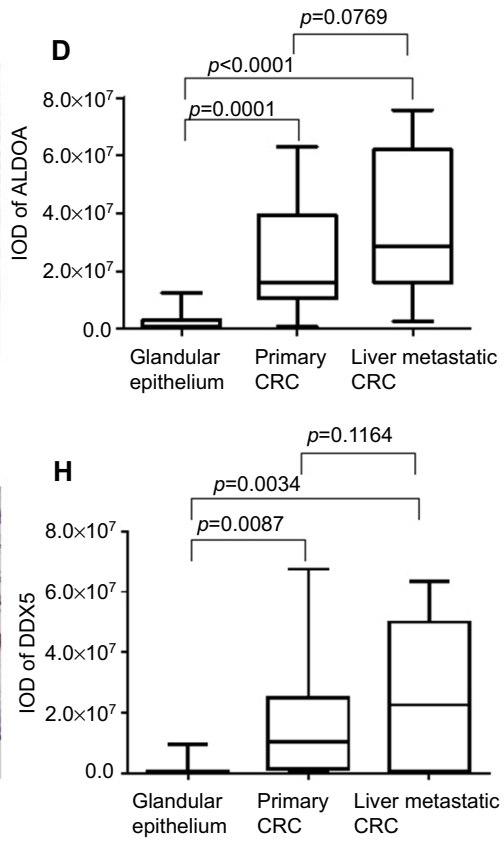

Figure I Expression of ALDOA and DDX5 in paired adjacent glandular epithelium, primary colorectal cancer, and liver metastatic colorectal cancer tissue.

Notes: Expression of ALDOA in (A) adjacent glandular epithelium $(\times 200)$, (B) primary colorectal cancer tissue $(\times 200)$, (C) liver metastatic colorectal cancer tissue $(\times 200)$. (D) Box plot showing the staining intensity (mean with SEM) of ALDOA in paired adjacent glandular epithelium, primary colorectal cancer, and liver metastatic colorectal cancer tissues. Expression of DDX5 in (E) adjacent glandular epithelium $(\times 200)$, (F) primary colorectal cancer tissue $(\times 200)$, (G) liver metastatic colorectal cancer tissue $(\times 200)$. (H) Box plot showing the staining intensity (mean with SEM) of ALDOA in paired adjacent glandular epithelium, primary colorectal cancer, and liver metastatic colorectal cancer tissues.

Abbreviations: ALDOA, fructose-bisphosphate aldolase A; CRC, colorectal cancer; DDX5, DEAD box p68 RNA helicase; IOD, integrated optical density. 
$\mathbf{A}$

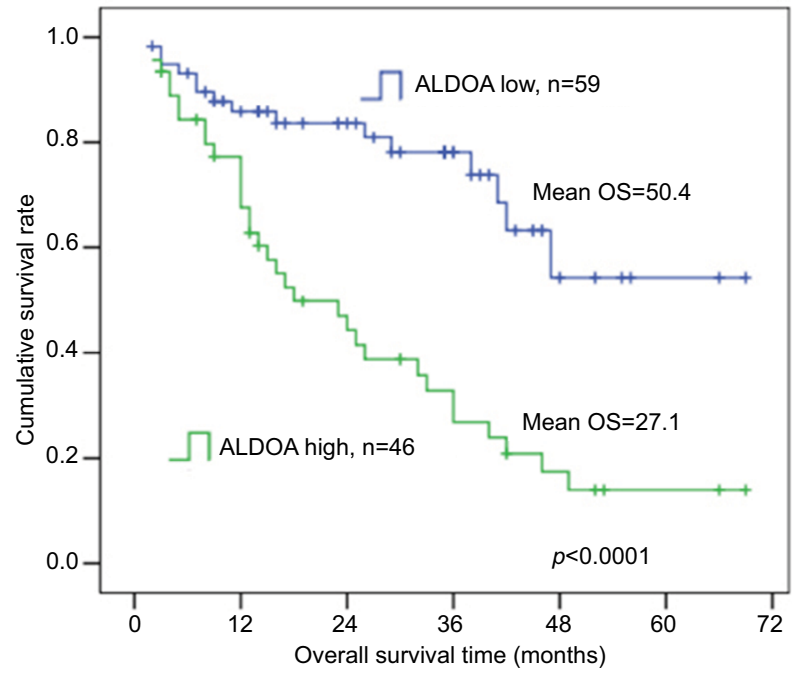

C

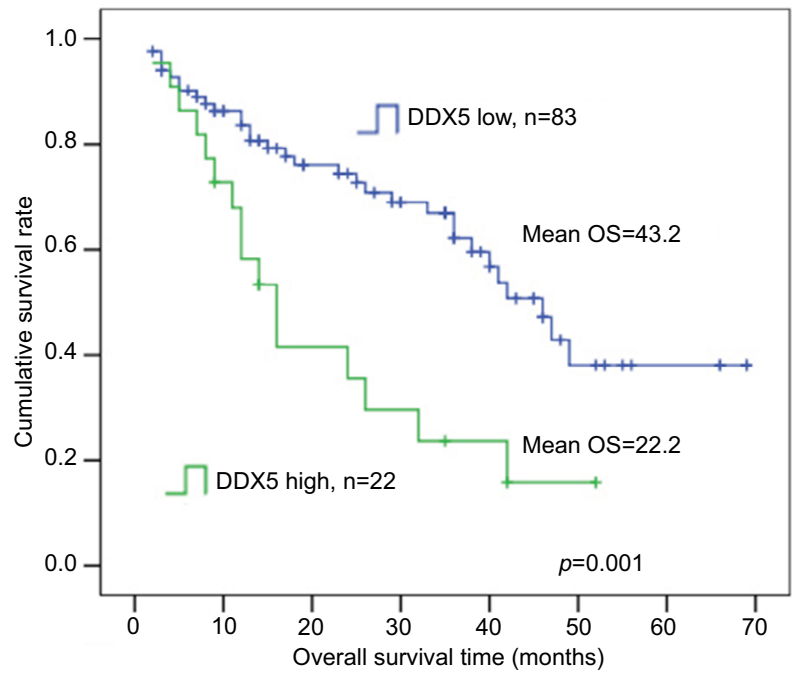

E

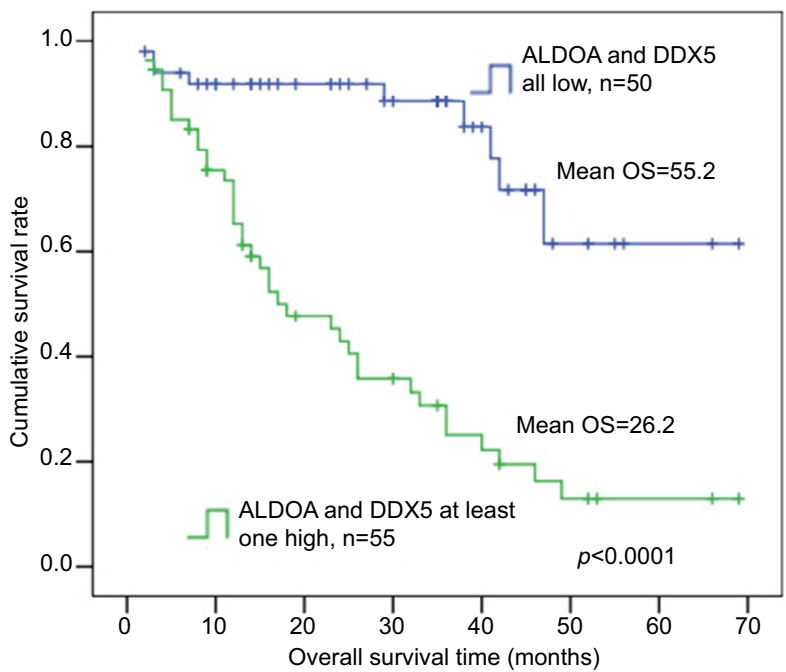

B

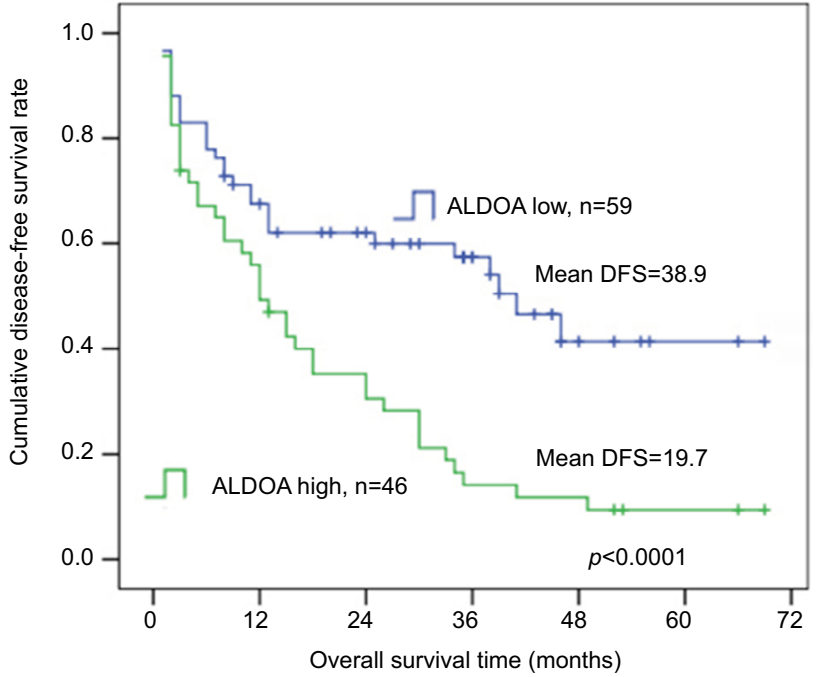

D

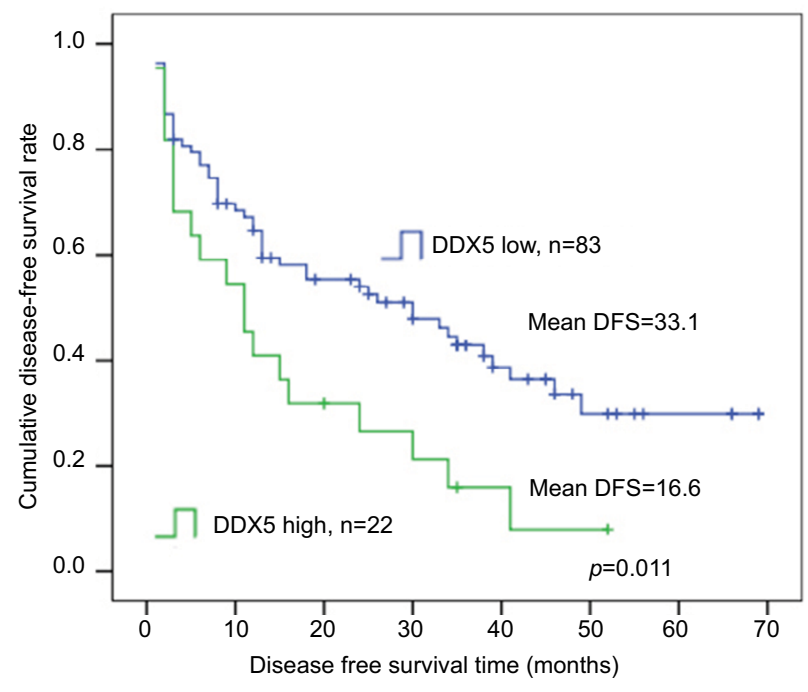

$\mathbf{F}$

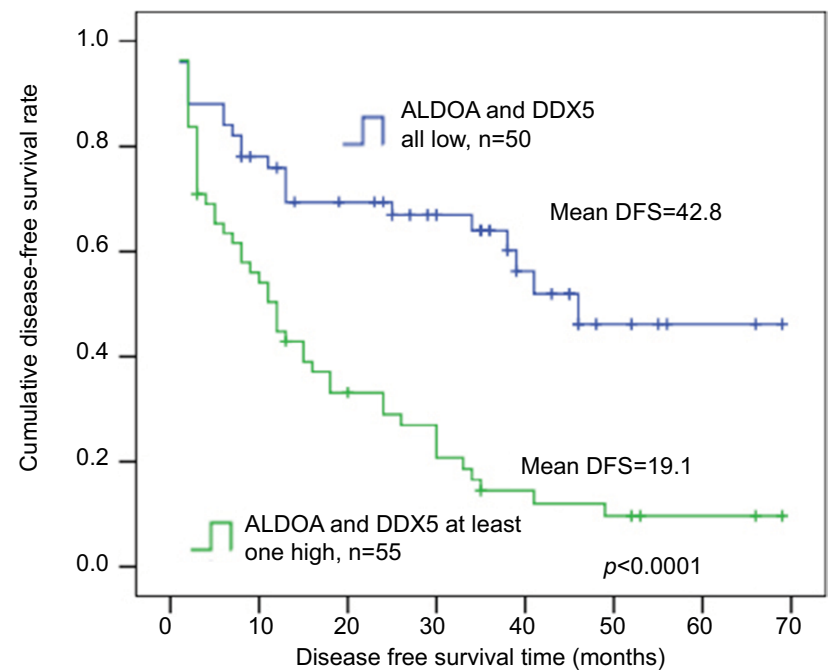

Figure 2 Positive correlation of high ALDOA expression and DDX5 high expression with poor OS and DFS in colorectal cancer patients.

Notes: Probabilities of (A) OS (high ALDOA=46, low ALDOA=59, $p<0.0001$ ) and (B) DFS ( $p<0.0001$ ) in colorectal cancer patients. Probabilities of (C) OS (high DDX5=22, low DDX5 $=83, p=0.001$ ) and (D) DFS $(p=0.01 \mathrm{I})$ in colorectal cancer patients. Probabilities of (E) OS (ALDOA and DDX5 all low, $n=50, A L D O A$ and DDX5 at least one high, $n=55, p<0.000 \mathrm{I})$ and $(\mathbf{F}) \mathrm{DFS}(\mathrm{p}<0.000 \mathrm{I})$ in colorectal cancer patients. Log-rank test was determined using Kaplan-Meier survival analyses.

Abbreviations: ALDOA, fructose-bisphosphate aldolase A; DDX5, DEAD box p68 RNA helicase; DFS, disease-free survival; OS, overall survival. 


\section{Association of ALDOA and DDX5 expression with clinicopathological features of CRC patients}

We further investigated the association between ALDOA and DDX5 expression and clinicopathological factors in the 105 CRC patients. X2 analyses showed that DDX5 was not associated with any clinicopathological factors, while ALDOA expression was associated with serum CA19-9 level in CRC patients $(p=0.027)$ but was not associated with sex, age, tumor differentiation, $\mathrm{T}$ stage, $\mathrm{N}$ stage, $\mathrm{M}$ stage, TNM stage, serum carcinoembryonic antigen (CEA), liver metastasis, or postoperative chemotherapy (Table 1).

\section{Univariate and multivariate Cox regression analysis of risk factors for CRC patients after surgery}

To determine independent prognostic factors for CRC patients after surgery, univariate and multivariate analyses were performed based on a Cox proportional hazard regression model. Clinical and pathological factors showing statistical significance in Cox univariate analyses were included in Cox multivariate analyses. Cox univariate analyses showed that M stage ( $p<0.0001$ for both OS and DFS), TNM stage ( $p=0.005$ for OS and $p<0.0001$ for DFS), serum CA19-9 ( $p<0.0001$ for OS, $p=0.001$ for DFS), liver metastasis $(p<0.0001$ for both OS and DFS), ALDOA expression ( $p<0.0001$ for OS, $p=0.001$ for DFS), DDX5 expression ( $p=0.002$ for OS, $p=0.015$ for DFS), and ALDOA/DDX5 combination $(p<0.0001$ for both OS and DFS) were prognostic factors, and serum CA19-9 ( $p=0.004$ for OS, $p=0.005$ for DFS), liver metastasis ( $p<0.0001$ for both OS and DFS), and ALDOA/DDX 5 combination ( $p<0.0001$ for OS) and ALDOA expression ( $p=0.001$ for DFS) were independent prognostic factors in CRC patients after surgery (Table 2).

\section{Discussion}

$\mathrm{CRC}$ is the most commonly diagnosed cancer and a major cause of global cancer death in both males and females. ${ }^{22}$ The TNM staging system is a conventional predictor of outcome among postoperative CRC patients. Despite continuous refinement of the system to indicate the extent of the disease and define prognosis, with the aim of guiding treatment, OS and DFS among postoperative CRC patients may vary considerably, even within the same tumor stage..$^{23}$ Therefore, the need for novel biomarkers, especially those which might reflect tumor features to more precisely stratify patients into different risk categories, is clearly warranted. ${ }^{24}$
Table I Relationship between ALDOA and DDX5 expression levels, and the clinicopathological features of CRC

\begin{tabular}{|c|c|c|c|c|c|c|}
\hline \multirow[t]{2}{*}{ Variable } & \multicolumn{2}{|c|}{ ALDOA } & \multirow[t]{2}{*}{$p$-value } & \multicolumn{2}{|c|}{ DDX5 } & \multirow[t]{2}{*}{$p$-value } \\
\hline & Low & High & & Low & High & \\
\hline Sex & & & 0.060 & & & 0.915 \\
\hline Male & 39 & 22 & & 48 & 13 & \\
\hline Female & 20 & 24 & & 35 & 9 & \\
\hline Age & & & 0.383 & & & 0.363 \\
\hline$\leq 59$ & 32 & 21 & & 40 & 13 & \\
\hline$>59$ & 27 & 25 & & 43 & 9 & \\
\hline Tumor differentiation & & & 0.722 & & & 0.466 \\
\hline Well & 12 & 8 & & 15 & 5 & \\
\hline Moderate & 45 & 35 & & 65 & 15 & \\
\hline Poor & 2 & 3 & & 3 & I & \\
\hline T stage & & & 0.333 & & & 0.841 \\
\hline TI & 2 & 0 & & 2 & 0 & \\
\hline $\mathrm{T} 2$ & 5 & 6 & & 8 & 3 & \\
\hline T3 & 44 & 30 & & 59 & 15 & \\
\hline $\mathrm{T} 4$ & 8 & 10 & & 14 & 4 & \\
\hline$N$ stage & & & 0.496 & & & 0.239 \\
\hline No & 32 & 28 & & 45 & 15 & \\
\hline $\mathrm{NI}-\mathrm{N} 2$ & 27 & 18 & & 38 & 7 & \\
\hline M stage & & & 0.364 & & & 0.084 \\
\hline Mo & 36 & 24 & & 51 & 9 & \\
\hline MI & 23 & 22 & & 32 & 13 & \\
\hline TNM stage & & & 0.420 & & & 0.436 \\
\hline 1 & 4 & 4 & & 7 & I & \\
\hline II & 17 & 13 & & 25 & 5 & \\
\hline III & 15 & 6 & & 18 & 3 & \\
\hline IV & 23 & 23 & & 33 & 13 & \\
\hline Serum CEA & & & 0.952 & & & 0.861 \\
\hline$\leq 5 \mathrm{ng} / \mathrm{mL}$ & 26 & 20 & & 36 & 10 & \\
\hline$>5 \mathrm{ng} / \mathrm{mL}$ & 33 & 26 & & 47 & 12 & \\
\hline Serum CAI9-9* & & & 0.027 & & & 0.737 \\
\hline$\leq 37 \mathrm{U} / \mathrm{mL}$ & 39 & 21 & & 48 & 12 & \\
\hline$>37 \mathrm{U} / \mathrm{mL}$ & 19 & 25 & & 34 & 10 & \\
\hline Liver metastasis & & & 0.159 & & & 0.704 \\
\hline No & 40 & 25 & & 55 & 10 & \\
\hline Yes & 19 & 21 & & 28 & 12 & \\
\hline Postoperative & & & 0.455 & & & 0.687 \\
\hline chemotherapy* & & & & & & \\
\hline No & 8 & 9 & & 14 & 3 & \\
\hline Yes & 31 & 23 & & 42 & 12 & \\
\hline
\end{tabular}

Notes: *We failed to obtain some data. Statistically significant values are shown in bold $(p<0.05)$.

Abbreviations: ALDOA, fructose-bisphosphate aldolase A; CA 19-9, carbohydrate antigen 19-9; CEA, carcinoembryonic antigen; CRC, colorectal cancer; DDX5 DEAD box 968 RNA helicase.

Although serum CEA and CA19-9 are elevated in patients with CRC, they have not have not been recommended as screening tests for CRC, due to their low sensitivity and specificity. Also, they were found to be significantly elevated in colorectal neoplasia. ${ }^{25-27}$ However, serum CEA and CA19-9 are recommended as a prognostic biomarker for monitoring recurrence of $\mathrm{CRC}$ following curative resection and predicting prognosis. ${ }^{25,28}$ Univariate analysis of the present study 
Table 2 Univariate and multivariate analyses of factors associated with OS and DFS in CRC patients

\begin{tabular}{|c|c|c|c|c|c|c|c|c|}
\hline Factors & Univariate $P$ & HR & $\begin{array}{l}\text { OS } \\
\text { Multivariate } \\
95 \% \mathrm{Cl}\end{array}$ & $p$-value & Univariate $P$ & HR & $\begin{array}{l}\text { DFS } \\
\text { Multivariate } \\
95 \% \mathrm{Cl}\end{array}$ & p-value \\
\hline Sex: male vs. female & 0.627 & & & & 0.624 & & & \\
\hline Age: $\leq 59$ vs. $>59$, years & 0.712 & & & & 0.572 & & & \\
\hline \multicolumn{9}{|l|}{ Tumor differentiation: } \\
\hline Well vs. moderate vs. poor & 0.079 & & & & 0.097 & & & \\
\hline T stage: $\mathrm{TI}$ vs. $\mathrm{T} 2$ vs. $\mathrm{T} 3$ vs. $\mathrm{T} 4$ & 0.530 & & & & 0.889 & & & \\
\hline N stage: N0 vs. NI-N2 & 0.852 & & & & 0.108 & & & \\
\hline M stage: $M 0$ vs. MI & $<0.000$ I & & & & $<0.0001$ & & & \\
\hline TNM stage: I vs. II vs. III vs. IV & 0.005 & & & & $<0.000$ I & & & \\
\hline Serum CEA (ng/mL): $\leq 5$ vs. $>5$ & 0.062 & & & & 0.073 & & & \\
\hline Serum CAI $9-9(\mathrm{U} / \mathrm{mL}): \leq 37$ vs. $>37$ & $<0.0001$ & 2.466 & $1.325-4.589$ & 0.004 & 0.001 & 2.090 & $1.243-3.513$ & 0.005 \\
\hline Liver metastasis: no vs. yes & $<0.0001$ & 4.807 & $2.398-9.635$ & $<0.0001$ & $<0.0001$ & 8.043 & $4.557-14.194$ & $<0.0001$ \\
\hline Postoperative chemotherapy: no vs. yes & 0.669 & & & & 0.072 & & & \\
\hline ALDOA: low vs. high & $<0.000$ I & & & & 0.001 & 2.145 & $1.291-3.562$ & 0.003 \\
\hline DDX5: low vs. high & 0.002 & & & & 0.015 & & & \\
\hline \multicolumn{9}{|l|}{ ALDOA/DDX5 combination: } \\
\hline All low vs. at least one high & $<0.0001$ & 5.322 & $2.434-11.634$ & $<0.000$ I & $<0.0001$ & & & \\
\hline
\end{tabular}

Note: Statistically significant values are shown in bold $(p<0.05)$.

Abbreviations: ALDOA, fructose-bisphosphate aldolase A; CAI9-9, carbohydrate antigen 19-9; CEA, carcinoembryonic antigen; CRC, colorectal cancer; DDX5, DEAD box p68 RNA helicase; DFS, disease-free survival; HR, hazard radio; OS, overall survival.

reveals that serum CEA had a tendency to predict prognosis and serum CA19-9 is an independent prognostic factor for both OS and DFS.

Immunohistochemical staining is widely used for visualizing these tumor markers and is a routine tool in the department of pathology. Although several studies have reported diagnostic and prognostic markers of CRC for immunohistochemistry assays, few are routinely used in clinical pathology. Thus, identification of a new molecular marker for use in immunohistochemistry of tumor tissues from CRC patients would be beneficial for effective individualized therapy for this disease.

In this present study, we initially evaluated the significance of ALDOA and DDX5 expressions in 18 paired normal glandular epithelium tissues, primary CRC tissues, and liver metastatic CRC tissues. The paired $t$-test revealed that ALDOA and DDX5 expression were significantly higher in primary CRC tissues and liver metastatic CRC tissues than in normal glandular epithelium tissues. Furthermore, ALDOA and DDX5 expression were slightly higher in liver metastatic CRC tissues than in primary tissues, although the small sample size meant that the difference was not statistically significant ( $p=0.0769$ for ALDOA and $p=0.1164$ for DDX5). Further studies in a larger sample of paired liver metastatic CRC and primary tissues are therefore warranted.

More importantly, Kaplan-Meier survival analyses clearly showed that patients with high ALDOA or high DDX5 in expression in primary CRC tissues had shorter
OS and DFS than patients with low ALDOA or low DDX5 expressions. Univariate and multivariate Cox regression analyses showed that serum CA19-9, liver metastasis, and ALDOA/DDX5 combination was an independent prognostic factor for OS, and serum CA19-9, liver metastasis, ALDOA were independent prognostic factors for DFS. Notably, multivariate Cox regression analyses showed that hazard ratio and $p$-value from multivariate Cox regression analyses reveals that ALDOA/DDX5 combination was more effective for predicting postoperative OS of CCR patients. Of note, dynamic proteomic analysis has recently identified ALDOA as a novel biomarker of CRC prognosis ${ }^{29}$ and DDX5 was reported as a transcriptional co-activator in tumor development; ${ }^{30,31}$ however, detailed information on the prognostic value of ALDOA and DDX5 and its expression characteristics in liver metastatic CRC tissues has not been reported.

To the best of our knowledge, our study is the first to demonstrate the prognostic value of ALDOA, DDX5, and ALDOA/ DDX5 combination as an independent prognostic factor for OS and DFS in CRC patients after surgery. Furthermore, ALDOA and or ALDOA/DDX 5 combined with serum CA19-9, liver metastasis may effectively predict outcome of CRC patients.

\section{Conclusion}

The present study clearly demonstrates that ALDOA and DDX5 protein are highly expressed in CRC tumor and liver metastatic CRC tissues compared with normal glandular epithelium tissues, and that increased expression of ALDOA 
and DDX5 in CRC tissues may indicate poor prognosis in patients after surgery. Therefore, our findings indicate that ALDOA and DDX5 are potential biomarkers for CRC, and may represent a useful approach to the prediction of OS and DFS in CRC patients after surgery.

\section{Acknowledgments}

This study was supported by the National Natural Science Foundation of China (81360326, 81260354, 81460458), Guangxi Natural Science Foundation of China (2014GXNSFAA118219, 2015GXNSFAA139157), Bagui Scholar Foundation of He Songqing, and the "Sphingolipids and Related Diseases" Programme for the Innovative Research Team of Guilin Medical University. We thank Clare Cox from Liwen Bianji, Edanz Group China for editing the English text of a draft of this manuscript.

\section{Author contributions}

All authors made substantial contributions to conception and design, acquisition of data, or analysis and interpretation of data; took part in drafting the article or revising it critically for important intellectual content; gave final approval of the version to be published; and agree to be accountable for all aspects of the work.

\section{Disclosure}

The authors report no conflicts of interest in this work.

\section{References}

1. Chen W, Zheng R, Baade PD, et al. Cancer statistics in China, 2015. CA Cancer J Clin. 2016;66(2):115-132.

2. Gibson TB, Ranganathan A, Grothey A. Randomized phase III trial results of panitumumab, a fully human anti-epidermal growth factor receptor monoclonal antibody, in metastatic colorectal cancer. Clin Colorectal Cancer. 2006;6(1):29-31.

3. Hurwitz HI, Fehrenbacher L, Hainsworth JD, et al. Bevacizumab in combination with fluorouracil and leucovorin: an active regimen for firstline metastatic colorectal cancer. J Clin Oncol. 2005;23(15):3502-3508.

4. Wolpin BM, Meyerhardt JA, Mamon HJ, Mayer RJ. Adjuvant treatment of colorectal cancer. CA Cancer J Clin. 2007;57(3):168-185.

5. Tokunaga R, Sakamoto Y, Nakagawa S, et al. Prognostic nutritional index predicts severe complications, recurrence, and poor prognosis in patients with colorectal cancer undergoing primary tumor resection. Dis Colon Rectum. 2015;58(11):1048-1057.

6. Sun HM, Mi YS, Yu FD, et al. SERPINA4 is a novel independent prognostic indicator and a potential therapeutic target for colorectal cancer. Am J Cancer Res. 2016;6(8):1636-1649.

7. Lee KS, Kwak Y, Nam KH, et al. Favorable prognosis in colorectal cancer patients with co-expression of c-MYC and ss-catenin. BMC Cancer. 2016;16(1):730.

8. Filella X, Molina R, Grau JJ, et al. Prognostic value of CA 19.9 levels in colorectal cancer. Ann Surg. 1992;216(1):55-59.

9. Esposito G, Vitagliano L, Costanzo P, et al. Human aldolase A natural mutants: relationship between flexibility of the $\mathrm{C}$-terminal region and enzyme function. Biochem J. 2004;380(Pt 1):51-56.
10. Kukita A, Yoshida MC, Fukushige S, et al. Molecular gene mapping of human aldolase A (ALDOA) gene to chromosome 16. Hum Genet. 1987;76(1):20-26.

11. Oparina NY, Snezhkina AV, Sadritdinova AF, et al. Differential expression of genes that encode glycolysis enzymes in kidney and lung cancer in humans. Genetika. 2013;49(7):814-823.

12. Lessa RC, Campos AH, Freitas CE, et al. Identification of upregulated genes in oral squamous cell carcinomas. Head Neck. 2013;35(10): 1475-1481.

13. Shimizu T, Inoue K, Hachiya H, Shibuya N, Shimoda M, Kubota K. Frequent alteration of the protein synthesis of enzymes for glucose metabolism in hepatocellular carcinomas. $J$ Gastroenterol. 2014;49(9):1324-1332.

14. Dai TY, Cao L, Yang ZC, et al. P68 RNA helicase as a molecular target for cancer therapy. J Exp Clin Cancer Res. 2014;33:64.

15. Singh C, Haines GK, Talamonti MS, Radosevich JA. Expression of p68 in human colon cancer. Tumour Biol. 1995;16(5):281-289.

16. Haines GK, Cajulis R, Hayden R, Duda R, Talamonti M, Radosevich JA. Expression of the double-stranded RNA-dependent protein kinase (p68) in human breast tissues. Tumour Biol. 1996;17(1):5-12.

17. Dosaka-Akita H, Harada M, Miyamoto H, Kawakami Y. Clinical significance of oncogene product expression in human lung cancer. Nihon Kyobu Shikkan Gakkai zasshi. 1992;30(8):1441-1447.

18. Wang SJ, Zhang C, You Y, Shi CM. Overexpression of RNA helicase p68 protein in cutaneous squamous cell carcinoma. Clin Exp Dermatol. 2012;37(8):882-888.

19. Fuller-Pace FV, Moore HC. RNA helicases p68 and p72: multifunctional proteins with important implications for cancer development. Future Oncol. 2011;7(2):239-251.

20. Tan N, Liu Q, Liu X, et al. Low expression of B-cell-associated protein 31 in human primary hepatocellular carcinoma correlates with poor prognosis. Histopathology. 2016;68(2):221-229.

21. Camp RL, Dolled-Filhart M, Rimm DL. X-tile: a new bio-informatics tool for biomarker assessment and outcome-based cut-point optimization. Clin Cancer Res. 2004;10(21):7252-7259.

22. Parkin DM, Bray F, Ferlay J, Pisani P. Global cancer statistics, 2002. CA Cancer J Clin. 2005;55(2):74-108.

23. Mao YP, Xie FY, Liu LZ, et al. Re-evaluation of 6th edition of AJCC staging system for nasopharyngeal carcinoma and proposed improvement based on magnetic resonance imaging. Int J Radiat Oncol Biol Phys. 2009;73(5):1326-1334.

24. Puppa G, Sonzogni A, Colombari R, Pelosi G. TNM staging system of colorectal carcinoma: a critical appraisal of challenging issues. Arch Pathol Lab Med. 2010;134(6):837-852.

25. Locker GY, Hamilton S, Harris J, et al. ASCO 2006 update of recommendations for the use of tumor markers in gastrointestinal cancer. J Clin Oncol. 2006;24(33):5313-5327.

26. Bagaria B, Sood S, Sharma R, Lalwani S. Comparative study of CEA and CA19-9 in esophageal, gastric and colon cancers individually and in combination (ROC curve analysis). Cancer Biol Med. 2013;10(3):148-157.

27. Kim NH, Lee MY, Park JH, et al. Serum CEA and CA 19-9 levels are associated with the presence and severity of colorectal neoplasia. Yonsei Med J. 2017;58(5):918-924.

28. Yu Z, Chen Z, Wu J, Li Z, Wu Y. Prognostic value of pretreatment serum carbohydrate antigen 19-9 level in patients with colorectal cancer: a meta-analysis. 2017;12(11):e0188139.

29. Peng Y, Li X, Wu M, et al. New prognosis biomarkers identified by dynamic proteomic analysis of colorectal cancer. Mol Biosyst. 2012;8(11):3077-3088.

30. Nicol SM, Fuller-Pace FV. Analysis of the RNA helicase p68 (Ddx5) as a transcriptional regulator. Methods Mol Biol. 2010;587:265-279.

31. Sambasivan R, Cheedipudi S, Pasupuleti N, Saleh A, Pavlath GK, Dhawan J. The small chromatin-binding protein $\mathrm{p} 8$ coordinates the association of anti-proliferative and pro-myogenic proteins at the myogenin promoter. J Cell Sci. 2009;122(Pt 19):3481-3491. 


\section{Publish your work in this journal}

Cancer Management and Research is an international, peer-reviewed open access journal focusing on cancer research and the optimal use of preventative and integrated treatment interventions to achieve improved outcomes, enhanced survival and quality of life for the cancer patient. The manuscript management system is completely online and includes a very quick and fair peer-review system, which is all easy to use. Visit http://www.dovepress.com/testimonials.php to read real quotes from published authors.

Submit your manuscript here: https://www.dovepress.com/cancer-management-and-research-journal 